\title{
Lifestyle Modification in Cardiac Patient in COVID 19 Pandemic Time
}

\author{
ARIFUL ISLAM JOARDER ${ }^{1}$, A.K.M. IMTIAZAKAND' ${ }^{1}$, SHARMINAHMED ${ }^{1}$, MD. AZHARUL ISLAM1, \\ SM EAR-E-MAHABUB ${ }^{1}$, DIPAL KRISHNAADHIKARY ${ }^{1}$, MD. MOSTASHIRUL HAQUE ${ }^{1}$, MOHAMMED RAYHAN \\ MASUM MANDAL ${ }^{1}$, MD. FAKHRUL ISLAM KHALED ${ }^{1}$, SHAH MD. ZAKIR HOSSAIN ${ }^{2}$, MD. HARISUL HOQUE ${ }^{1}$ \\ ${ }^{1}$ Department of Cardiology, Bangabandhu Sheikh Mujib Medical University, Dhaka, ${ }^{2}$ Department of Nephrology, \\ Bangabandhu Sheikh Mujib Medical University, Dhaka
}

Address of Correspondence: Md. Fakhrul Islam Khaled, Assistant Professor, Department of Cardiology, Bangabandhu Sheikh Mujib Medical University, Dhaka, email: drfikhaled@gmail.com

University Heart Journal 2020; 16(2): 111-114

\begin{abstract}
Introduction:
Severe acute respiratory syndrome coronavirus 2 (SARSCoV 2) causing coronavirus disease 2019 (COVID-19) has reached pandemic levels; patients with cardiovascular (CV) risk factors and established cardiovascular disease (CVD) represent a vulnerable population when suffering from COVID-19;Patients with cardiac injury in the context of COVID-19 have an increased risk of morbidity and mortality.
\end{abstract}

The SARS-CoV 2 causing COVID-19 has reached pandemic levels since March 2020. In the absence of vaccines or curative medical treatment, COVID-19 exerts an unprecedented global impact on public health and health care delivery. Owing to the unexpected need for large capacities of intensive care unit (ICU) beds with the ability to provide respiratory support and mechanical ventilation, temporary redistribution and reorganization of resources within hospitals have become necessary with relevant consequences for all medical specialties. In addition, protective measures against SARS-CoV 2 gain particular significance for health care personnel (HCP) in direct contact with patients suffering from COVID-19 as well as for ambulatory and hospitalized patients without infection. In view of finite health care resources, health care providers are confronted with ethical considerations on how to prioritize access to care for individual patients as well as providing care for COVID-19 while not neglecting other life-threatening emergencies. Of note, assays to detect the virus in asymptomatic and symptomatic patients have important limitations in terms of sensitivity and specificity and will be complemented by tests for antibodies to identify those that already have been infected previously.

SARS-CoV 2 not only causes viral pneumonia but has major implications for the $\mathrm{CV}$ system. Patients with $\mathrm{CV}$ risk factors including male sex, advanced age, diabetes, hypertension and obesity as well as patients with established CV and cerebrovascular disease have been identified as particularly vulnerable populations with increased morbidity and mortality when suffering from COVID-19. Moreover, a considerable proportion of patients may develop cardiac injury in the context of COVID-19 which portends an increased risk of in-hospital mortality. Aside from arterial and venous thrombotic complications presenting as acute coronary syndromes (ACS) and venous thromboembolism (VTE), myocarditis plays an important role in patients with acute heart failure (HF).

\section{COVID-19 and cardiac arrhythmia}

Viral infections are associated with metabolic dysfunction, myocardial inflammation, and activation of the sympathetic nervous system, all of which predispose to cardiac arrhythmia. In a recent report on 138 hospitalized COVID19 patients, $16.7 \%$ of patients developed arrhythmias, which ranked only second among serious complications after ARDS.21

Arrhythmia was observed in $7 \%$ of patients who did not require ICU treatment and in $44 \%$ of subjects who were admitted to an ICU.18

\section{COVID-19 and myocardial injury and heart failure}

Most reports indicate that almost all hospitalized COVID19 patients show elevated serum creatine kinase $(\mathrm{CK})$ and lactate dehydrogenase (LDH) levels.6,43,44

In addition, a number of studies indicate that cardiac complications, including fulminant myocarditis, are potential outcomes of SARS-CoV-2 infection.

Heart failure has been reported as an outcome in $23 \%$ of COVID subjects in a recent report from in-hospital Chinese subjects.

Approximately $52 \%$ of non-survivors had heart failure as compared with $12 \%$ of survivors. 32 
Evidence of myocardial injury, such as an increase in highsensitivity cardiac troponin I (cTnI) levels ( $>28$

$\mathrm{pg} / \mathrm{mL}$ ) was detected in 5 of the first 41 patients diagnosed with COVID-19 in Wuhan.6,43,44

More recent reports indicate that $7.2 \% 21$ to $17 \% 32$ of hospitalized COVID-19 patients sustain acute myocardial injury.

This may be in the form of acute myocarditis (see below) or injury secondary to an oxygen supply/demand mismatch [type 2 myocardial infarction (MI)]

\section{COVID-19 and myocarditis}

Cardiac injury and acute myocarditis are well-recognized complications of acute viral infections. Myocyte necrosis and mononuclear cell infiltrates are reported in cardiac muscle autopsy.So, myocarditis may be an important cause of the acute cardiac injury in COVID-19 patients.2346,48

\section{COVID-19 and ischaemic heart disease}

Several pathways associated with viral diseases may contribute to destabilize plaques in COVID-19 patients.57

Heart failure patients are at increased risk of acute events or exacerbation; viral illness can potentially destabilize atherosclerotic plaques through systemic inflammatory responses, 58 cytokine storm, as well as specific changes of immune cell polarization towards more unstable phenotypes. All of these have been observed in COVID-19.

In the case of SARS and MERS, acute MI has been reported in two out of the five deaths in early reports.59.6061

It is important to consider that type $2 \mathrm{MI}$ (Hypoxia induced demand-supply mismatch) is the most common subtype in viral conditions.

Thus the usefulness of invasive management with a view toward coronary revascularization (especially in type 2 MI) is limited.

\section{COVID-19 and coagulation abnormalities}

Features of disseminated intravascular coagulation (DIC) and pulmonary embolism, characterized by increased Ddimer levels and fibrin degradation products, are highly prevalent in COVID-19. DIC has been observed in $71.4 \%$ of non-survivors. 66

Massive pulmonary embolism has been reported.67

Although early appearance of DIC features is often evident.

\section{Risk factors for cardiovascular disease}

Adults who fare poorly with COVID-19 (SARS-CoV-

2) infection more commonly have risk factors for (e.g., high blood pressure, obesity and diabetes) and/or known cardiovascular disease. Moreover, while it is especially important to lead a healthy lifestyle and manage chronic medical conditions during COVID-19, traditional outpatient care services have been significantly disrupted. Many patients are delaying or deferring necessary care, including preventive care. In addition, social distancing has negatively altered physical activity and eating habits in many adults and is contributing to higher-than-usual stress levels. To make things worse, virtually all facility-based cardiac rehabilitation programs for those who have recently experienced a cardiac event (e.g., heart attack, angioplasty/ stent, bypass surgery) have temporarily closed. If not promptly addressed, such changes are likely to adversely impact cardiovascular health. ${ }^{2}$ Many older adults fearful of COVID-19 are strictly adherent to social isolation measures and may be avoiding routine medical care. Continued management of cardiac disease during the pandemic is essential as optimization of cardiovascular health may not only decrease the risk of a cardiac admission but potentially mitigate vulnerability if exposed to SARS-CoV-2.

Encounters with noninfected older adults during the pandemic should focus on disease prevention and COVID19 preparation. Older adults with cardiovascular disease are at risk for both severe presentation of COVID-19, and lack of adequate cardiovascular disease monitoring during the pandemic. ${ }^{3}$ A Community-Based Cohort Study of 387,109 Adults in UK was done onLifestyle Risk Factors for Cardiovascular Disease in Relation to COVID-19 Hospitalization. There were 760 COVID-19 cases. After adjustment for age, sex and mutually for each lifestyle factor, physical inactivity (Relative risk, 1.32, 95\% confidence interval, 1.10, 1.58), smoking $(1.42 ; 1.12,1.79)$ and obesity $(2.05 ; 1.68,2.49)$ but not heavy alcohol consumption $(1.12 ; 0.93,1.35)$ were all related to COVID19. They also found a dose-dependent increase in risk of COVID-19 with less favourable lifestyle scores, such that participants in the most adverse category had 4-fold higher risk $(4.41 ; 2.52-7.71)$ compared to people with the most optimal lifestyle. This gradient was little affected after adjustment for a wide range of covariates. Based on UK risk factor prevalence estimates, unhealthy behaviours in combination accounted for up to $51 \%$ of the population attributable fraction of severe COVID-19. ${ }^{4}$

lifestyle modifications that lower risk factors for heart and vascular disease, prolonging and improving quality of life. The key is to know risk factors and to modify them.

\section{Risk Factors that can be Controled}

While there are risk factors that cannot be changed, there are some that can be controled over and some that can be 
influenced. Here's an overview of the risk factors we may be able to impact by following some guidelines for hearthealthy living:

Smoking and using other tobacco products Smoking expose heart and blood vessels to nicotine, carbon monoxide and other harmful substances contained in smoke. This causes blood vessels to constrict, blood pressure to go up and cholesterol levels to climb. In addition, smoking deprives the body's tissues of oxygen, damages the inner lining of blood vessels, allows plaques to grow inside your arteries, and makes it more likely that dangerous blood clots will form.

High cholesterol levels. High levels of LDL cholesterolthe so-called bad cholesterol - can increase the build-up of plaque in the arteries of the heart. It's also unhealthy to have low levels of HDL, or "good" cholesterol. The ideal blood cholesterol level depends on your age, gender, and history of heart disease, but for most people with coronary artery disease, the target LDL cholesterol level is $100 \mathrm{mg}$ / $\mathrm{dL}$ or below, and the target HDL cholesterol level is above $40 \mathrm{mg} / \mathrm{dL}$ for men, and above $50 \mathrm{mg} / \mathrm{dL}$ for women.

High blood pressure.If blood pressure is above 140/90 $\mathrm{mmHg}$ for long periods of time, it can damage the blood vessels. This not only makes it more likely that cholesterol plaques will form, it also causes the artery walls to become thicker, stiffer and less able to expand and contract with changes in activity and other physical demands.

Diabetes. High blood sugar levels can damage the blood vessels throughout the body, making it more likely that atherosclerotic plaques will develop. In fact, about one out of three people with diabetes also has coronary artery disease.

Being overweight or obese. Carrying around too much body weight not only puts a strain on heart, it also makes it more difficult to control high blood pressure, high cholesterol levels, and diabetes. Men and women who are overweight are about 20 percent more likely to develop cardiovascular disease. Being obese-more than 20 percent above ideal body weight - is even more dangerous. Obesity increases the risk of cardiovascular disease by 46 percent in men and 64 percent in women.

Physical inactivity. A lack of exercise weakens muscles and makes it harder to control several other heart disease risk factors, including blood pressure, cholesterol levels, diabetes, obesity, and stress.

Metabolic syndrome. The term metabolic syndrome is used to describe a cluster of traits that, together, increase the risk for developing heart disease. These traits include high blood sugar, high blood pressure, low levels of "good" HDL cholesterol, high blood levels of fats known as triglycerides, and excess body weight, particularly in the belly area.

Stress. High levels of stress in life, or a tendency to often feel angry, have also been linked to an increased risk for heart disease. Learning how to manage stress can be good for your heart.

High levels of C-reactive protein. CRP is produced by the body in response to infection or inflammation. special highsensitivity CRP test to look for signs of inflammation in the arteries of heart. If CRP levels are high, risk of having a heart attack is increased.

\section{Lifestyle Changes for Heart and Vascular Disease}

Get proper nutrition: Limit your intake of fat, cholesterol and sodium.

- Exercise regularly: Talk with your care team about starting an exercise program or joining a formal cardiac rehabilitation program. After a hospital stay, slowly increase your activities each day.

- Manage obesity: If you are overweight, talk with your care team about a weight-loss program, including diet, exercise and counseling.

- Minimize or eliminate tobacco and alcohol: Stop smoking and drink alcohol sparingly, if at all.

- Control diabetes: If you have diabetes, you are at least twice as likely to have cardiovascular disease or a stroke.

- Treat sleep apnea: Obstructive sleep apnea increases your risk of hypertension (high blood pressure).

- Stay current on vaccinations: Talk to your care team about influenza and pneumococcal vaccinations.

Special lifestyle modifications

- Heart failure: If you have heart failure, weigh yourself every morning. Rapid weight gain is a heart failure warning sign.

- Arrhythmia: Stress, caffeine and alcohol can promote arrhythmia, so your care team may recommend lifestyle changes to help you reduce your stress levels. Your treatment plan may also include efforts to minimize or eliminate caffeinated beverages, tobacco and alcohol.

\section{Conclusion:}

An unhealthy lifestyle synonymous with an elevated risk of non-communicable disease is also a risk factor for 
COVID-19 hospital admission, accounting for up to half of severe cases. Adopting simple lifestyle changes could lower the risk of severe infection

\section{References:}

1. ESC Guidance for the Diagnosis and Management of CV Disease during the COVID-19 Pandemic. https:// www.escardio.org/Education/COVID-19-and-Cardiology/ESCCOVID-19-Guidance. (Last update:10 June 2020)

2. 1.Colpani V, Baena CP, Jaspers L, van Dijk GM, Farajzadegan Z, Dhana K, Tielemans MJ, Voortman T, Freak-Poli R, Veloso GGV, Chowdhury R, Kavousi M, Muka T, Franco OH. Lifestyle factors, cardiovascular disease and all-cause mortality in middle-aged and elderly women: a systematic review and metaanalysis. Eur J Epidemiol. 2018 Sep;33(9):831-845.

3. Schlesinger S, Neuenschwander M, Ballon A, Nöthlings U, Barbaresko J. Adherence to healthy lifestyles and incidence of diabetes and mortality among individuals with diabetes: a systematic review and meta-analysis of prospective studies. J Epidemiol Community Health. 2020 May;74(5):481-487.

4. Nyberg ST, Singh-Manoux A, Pentti J, Madsen IEH, Sabia S, Alfredsson L, Bjorner JB, Borritz M, Burr H, Goldberg M,
Heikkilä K, Jokela M, Knutsson A, Lallukka T, Lindbohm JV, Nielsen ML, Nordin M, Oksanen T, Pejtersen JH, Rahkonen O, Rugulies R, Shipley MJ, Sipilä PN, Stenholm S, Suominen S, Vahtera J, Virtanen M, Westerlund H, Zins M, Hamer M, Batty GD, Kivimäki M.Association of Healthy Lifestyle With Years Lived Without Major Chronic Diseases. JAMA Intern Med. 2020 Apr 6. doi: 10.1001/jamainternmed.2020.0618. [Epub ahead of print]

5. Li Y, Schoufour J, Wang DD, Dhana K, Pan A, Liu X, Song M, Liu G, Shin HJ, Sun Q, Al-Shaar L, Wang M, Rimm EB, Hertzmark E, Stampfer MJ, Willett WC, Franco OH, $\mathrm{Hu}$ FB.Healthy lifestyle and life expectancy free of cancer, cardiovascular disease, and type 2 diabetes: prospective cohort study. BMJ. 2020 Jan 8;368:16669. doi: 10.1136/bmj.16669.

6. Guan WJ, Ni ZY, Hu Y, Liang WH, Ou CQ, He JX, et al.; China Medical Treatment Expert Group for Covid-19. Clinical characteristics of coronavirus disease 2019 in China. N Engl J Med. 2020 Apr;382(18):1708-20.

7. WHO. Coronavirus disease 2019 (COVID-19): situation report, 59. 2020. Available from: https://apps.who.int/iris/ handle/10665/331597. 